\title{
Retinovascular findings in newborns with critical congenital heart disease: A case series
}

\author{
Nurdan Fettah, M.D. ${ }^{a}$, Emrah U. Kabatas, M.D. ${ }^{b}$, Vehbi Doğan, M.D. ${ }^{c}$, Aysegül Zenciroğlu, M.D., Assoc. Prof..$^{a}$, \\ Dilek Dilli, M.D., Assoc. Prof. ${ }^{a}$, Elif Özyazıcı, M.D. ${ }^{a}$, Murat Koç, M.D. ${ }^{d}$, Serdar Beken, M.D. Assoc. Prof. ${ }^{a}$, \\ Arzu Dursun, M.D., Assoc. Prof. ${ }^{a}$ and Selmin Karademir, M.D.
}

\begin{abstract}
Critical congenital heart disease (CCHD) may cause changes in retinal vasculature. However, there is lack of data in this issue in newborns. We evaluated retinovascular changes in a series of 43 newborn with CCHD. They were divided into 2 groups according to the type of CCHD; group $1(\mathrm{n}=18)$ : left obstructive CCHD and group $2(\mathrm{n}=25)$ : right obstructive CCHD. Retinovascular pathologies were detected in 21 patients $(48.8 \%)$; it was more frequent in group $1(\mathrm{p}=0.04)$. The most common pathology was retinal vascular tortuosity in 6 patients $(33.3 \%)$ of group 1, and $4(16.0 \%)$ in group 2. None of 21 patients with retinovascular changes required any therapy at follow-up. In multivariate analysis, only having a left obstructive CCHD was associated with the development of any retinovascular pathology $(\mathrm{P}=0.03$, OR: 2.8, CI95\%: 1.1-7.4). Retinovascular changes are frequent in newborn patients with CCHD.

Key words: congenital heart defects, retinal vessels abnormalities, newborn.
\end{abstract}

http:/ / dx.doi.org/10.5546/ aap.2017.eng.e175

To cite: Fettah N, Kabatas EU, Doğan V, et al. Retinovascular findings in newborns with critical congenital heart disease: A case series. Arch Argent Pediatr 2017;115(3):e175-e178.

\section{INTRODUCTION}

Congenital heart disease (CHD) is an abnormal cardiocirculatory structure or function present at birth, although the disease is generally diagnosed at postnatal period. Both environmental factors

a Department of Neonatology.

b. Department of Ophtalmology.

c. Department of Pediatric Cardiology.

d. Department of Pediatric Cardiovascular Surgery,

Dr. Sami Ulus Maternity and Children Research and

Training Hospital, Ankara, Turkey.

E-mail Address:

Dilek Dilli, M.D.: dilekdilli2@yahoo.com

Funding: None.

Conflict of interest: None.

Received: 10-2-2016

Accepted: 12-27-2016 and genetic factors have been implicated in the etiopathogenesis of CHD. ${ }^{1}$ It has been reported that CHD affects 8 out of every 1,000 newborns. ${ }^{2}$ About $25 \%$ of babies with CHD have a critical $\mathrm{CHD}$ (CCHD) that often need surgery or other procedures in the first year of life., ${ }^{2,3}$

Numerous reports have shown that CHD may have effects on the retinal vasculature..$^{4-6}$ To the best of our knowledge, there is no investigations about the retinovascular changes in newborns with CCHD. Our aim was to determine retinovascular findings in newborns with CCHD.

\section{MATERIAL AND METHODS}

In this case series, we included 43 term or near-term newborn patients with CCHD admitted to a tertiary NICU, between May 2013 and December 2014. Infants whose gestational age was $<35$ weeks and with life major congenital abnormality were excluded. The infants without parental consent were not included. The diagnosis of CCHD was established by echocardiography or cardiac catheterization performed by a pediatric cardiologist. CCHD was defined as a $\mathrm{CHD}$ requiring surgical or transcatheter cardiac intervention. Intervention risk score according to Risk Adjustment in Congenital Heart Surgery (RACHS-1) classification was evaluated. ${ }^{7}$

Eye examinations were performed by the same ophthalmologist within the first week of NICU admission. The eye examinations were repeated weekly when any retinovascular changes were determined. Delayed retinal vascularization was defined as the disadvancement of retinal vessels reaching to temporal ora serrata beyond $40 \mathrm{wk}$ of gestational age.

The lowest and the highest hematocrit concentrations (Hct.; \%), the lowest and the highest partial arterial oxygen pressures $\left(\mathrm{PaO}_{2}\right.$; $\mathrm{mmHg}$ ) obtained from arterial blood gas tests and the number of red blood cell (RBC) transfusions were recorded. The durations of mechanical ventilation, nasal continue positive airway pressure (CPAP), free oxygen and total oxygen exposure times were registered. 
The patients were divided into two groups; group 1: left obstructive CCHD and group 2: right obstructive CCHD. Groups were compared in terms of clinical features and retinovascular findings.

The Local Ethics Committee approved the study. All parents were fully informed about the nature of this study as well as its aim. A written consent was obtained from all parents.

\section{Statistical analyses}

Data were analyzed by a computer using SPSS 18.0 statistics package program (SPSS Inc. Chicago, Illinois, USA). Parametric data were represented as mean \pm standard deviation (SD) and nonparametric data as the median and interquartile range (IQR). Differences between the groups were tested using Student's $t$ test or Mann Whitney U test, as appropriate. Chi-square test was used for categorical data. Multivariate analysis was used to define the factors associated with the presence of any retinovascular pathology in patients with CCHD. Gestational age, the type of CCHD, total oxygen exposure time, the number of RBC transfusion, the highest and the lowest Hct. and $\mathrm{PaO}_{2}$ levels were assigned as independent variables. The level of significance was set at 0.05 in all comparisons.

\section{RESULTS}

For all patients, the mean gestational age and birth weight were $38.0 \pm 1.4$ weeks and $3134 \pm 570 \mathrm{~g}$, respectively. Group 1 included 18 patients: aortic stenosis / atresia, $\mathrm{n}=3$; interrupted aortic arch, $\mathrm{n}=2$; aortic coarctation, $\mathrm{n}=11$; hypoplastic left heart syndrome, $\mathrm{n}=2$. There were 25 patients in group 2: pulmonary stenosis / atresia, $\mathrm{n}=23$; tetralogy of Fallot (TOF) $\mathrm{n}=2$. Demographic and clinical characteristics of the study patients by groups are seen in Table 1 .

Any retinovascular pathology was present in 21 patients $(48.8 \%)$, being more frequent in group $1(\mathrm{P}=0.04)$. Retinal vascular tortuosity was the most common pathology observed in 6 patients $(33.3 \%)$ in group 1 , and $4(16 \%)$ in group 2 (Figure 1). Delayed vascularization of the

TABLE 1. The demographical and clinical characteristics of the study patients

\begin{tabular}{|c|c|c|}
\hline & \multicolumn{2}{|c|}{$N=43$} \\
\hline & Group $1(n=18)$ & Group $2(n=25)$ \\
\hline Gestational age (weeks), mean \pm SD & $38,4 \pm 1,4$ & $37,7 \pm 1,3$ \\
\hline Birthweight $(\mathrm{g})$, mean $\pm \mathrm{SD}$ & $3332 \pm 366$ & $2992 \pm 650$ \\
\hline Cesarean rate, $\mathrm{n}(\%)$ & $8(53.3)$ & $7(28)$ \\
\hline Male sex, n (\%) & $11(64.7)$ & $14(56)$ \\
\hline Prenatal diagnosis, $\mathrm{n}(\%)$ & $1(5.6)$ & $3(12.0)$ \\
\hline Age at admission, median (IQR) & $6(3-9.5)$ & $6(1.5-10)$ \\
\hline RACHS-1 score, median (IQR) & $2(2-6)$ & $3(2-3)$ \\
\hline Retinovascular pathology, $\mathrm{n}(\%)$ & $12(66.7)$ & $9(36)$ \\
\hline Delayed vascularization & $2(11.1)$ & $5(20)$ \\
\hline Increased retinal vascular tortuosity & $6(33.3)$ & $4(16)$ \\
\hline Retinal hemorrhage & $1(5.6)$ & 0 \\
\hline Retinopathy of prematurity & $3(16.7)$ & 0 \\
\hline Total oxygen exposure time (day), median (IQR) & $23(18-33)$ & $25(12-49)$ \\
\hline Mechanical ventilation & $7(4.5-21)$ & $15(5-23)$ \\
\hline Nasal CPAP & $4(2.5-5.5)$ & $5(4-14)$ \\
\hline Free oxygen & $10(7-15)$ & $10.5(4-24)$ \\
\hline The number of red blood transfusion, median (IQR) & $2(1-3)$ & $3(1-4)$ \\
\hline \multicolumn{3}{|l|}{$\mathrm{PaO}_{2}(\mathrm{mmHg})$, median $(\mathrm{IQR})$} \\
\hline The highest $\mathrm{PaO}_{2}$ & $85(72-91)$ & $85(76-91)$ \\
\hline The lowest $\mathrm{PaO}_{2}^{2}$ & $43(32-58)$ & $40(35-55)$ \\
\hline \multicolumn{3}{|l|}{ Hct. $(\%)$, median (IQR) ${ }^{2}$} \\
\hline The highest $\mathrm{PaO}_{2}$ & $45(40-48)$ & $45(41-49)$ \\
\hline The lowest $\mathrm{PaO}_{2}$ & $30(27-33)$ & $29(28-34)$ \\
\hline Length of hospital stay (day), median (IQR) & $28(20-40)$ & $33(26-90)$ \\
\hline Mortality, $\mathrm{n}(\%)$ & $8(44.4)$ & $7(28.0)$ \\
\hline Chromosome 9 inversion & $1(5.6)$ & - \\
\hline Monosomy X & - & $1(0.4)$ \\
\hline
\end{tabular}

Group 1: Left obstructive critical congenital heart disease. Group 2: Right obstructive critical congenital heart disease. Hct.: Hematocrit. $\mathrm{PaO}_{2}$ : Partial arterial oxygen pressure. RACHS-1: Risk Adjustment in Congenital Heart Surgery. 
temporal retina was noted in 2 patients $(11.1 \%)$ in group 1 , and $5(20.0 \%)$ in group 2 . The mean gestational age of these infants were $37.1 \pm 0.9 \mathrm{wk}$. Following weekly eye examinations, completed retinal vascularization was determined at the mean postconceptional age of $46.3 \pm 1.6 \mathrm{wk}$.

In group 1, stage 1 ROP was detected in 3 near-term patients with aortic coarctation; the mean gestational age and birth weight were $36.6 \pm 0.5 \mathrm{wk}$ and $3463 \pm 686 \mathrm{~g}$, respectively. All 3 patients were inborn and admitted to NICU just after birth. Therefore their first eye examinations were performed within the first week of life. Retinopathy was determined at the second eye examinations performed one week later from the first exam. In the same group, retinal haemorrhage was detected in 1 patient $(5.6 \%)$, who also had a grade 2 intracranial haemorrhage. In multivariate analysis, only having a left obstructive CCHD was associated to the presence of any retinovascular pathology ( $\mathrm{P}=0.03$, OR: 2.8, CI 95\%:1.1-7.4).

Cytogenetic evaluation Giemsa banding (GTG) revealed monosomy $X$ in a girl patient with aortic coarctation who had phenotypic findings of Turner syndrome. Coarctation repair with resection and end-to-end anastomosis was performed. Chromosome 9 inversion was detected in two patients with pulmonary atresia. These patients were born to nonconsanguineous parents and had no other clinical abnormalities. They underwent Blalock Taussig (BT) shunt.

FIGURE 1. Increased retinal vascular tortuosity in a patient with aortic coarctation.

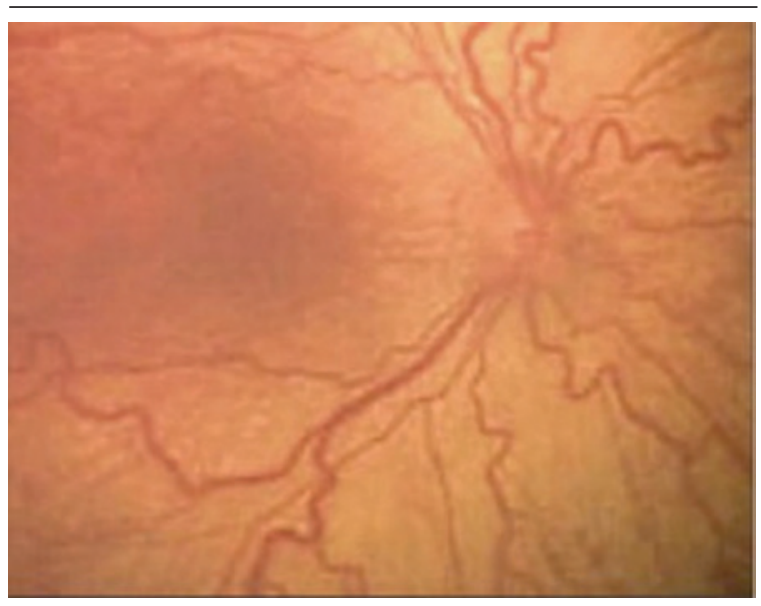

BT shunt was performed in 23 patients (53.5\%), coarctation repair with resection and end-to-end anastomosis in $13(30.2 \%)$, balloon valvuloplasty in $3(7.0 \%)$, Norwood operation in $2(4.7 \%)$, and radiofrequency ablation in $2(4.7 \%)$. Overall mortality rate was $34.9 \%(n=15)$. Seven of the 21 patients $(33.3 \%$ ) with retinovascular pathology died of underlying CCHD or its complications. Among survived infants, retinopathy and retinal hemorrhage were regressed spontaneously within five weeks after the first detection. Retinal vascular tortuosity was resolved nearly three months after the cardiac surgery.

\section{DISCUSSION}

In this case series, we observed that newborns with CCHD show various retinovascular pathologies. Although it is unclear what the exact mechanism is for these changes, it is suggested that most likely, a complex interplay of factors including increased viscosity and hypoxemia are involved in the pathogenesis. ${ }^{8,9}$

The mostly reported ocular findings in patients with cyanotic CHD are retinal vascular tortuosity, retinal haemorrhage, disk edema, papilledema, ischemic retinopathy, uveitis and central retinal vein occlusion. ${ }^{4,6}$ The other case reports have noted various vascular changes and retinopathy in cyanotic CHD. ${ }^{10-12}$ Of these, retinal vascular tortuosity appears to be the most frequent ophthalmologic change observed in patients with CHD as was in our case series.

The most important risk factors for the development of retinopathy of prematurity are low gestational age $(<32$ weeks) and low birth weight $(<1500 \mathrm{~g}) \cdot{ }^{13}$ However, stage 1 retinopathy was detected in three near-term patients with aortic coarctation. Aort coarctation may contribute to retinopathy development in near term or term patients even in the first weeks of life.

Nasal retina is normally fully vascularized after 8 months of gestation and the temporal periphery at or by 1 month after delivery in term healthy infants. ${ }^{13}$ Here, delayed vascularization of the temporal retina was noted in 7 patients. It may be considered that CHD might cause a delay the retinovascular maturation.

Peterson and Rosenthal ${ }^{14}$ reported that the severity of the fundus changes were associated to the patient's arterial oxygen saturation and Hct. Similarly, Mansour et al. ${ }^{4}$ have identified retinal vascular dilation and increased vascular tortuosity in patients with CHD. In our study, multivariate analysis showed that only having 
a left obstructive CCHD was associated with the development of any retinovascular pathology.

Tsui et al., ${ }^{10}$ described increased retinal vascular tortuosity in four asymptomatic adults with cyanotic CHD. They observed that a rise in the oxygen saturation levels after surgery has decreased the hypoxemia and erythrocytosis, with a normalization of the retinal vascularity patterns. Similarly we observed that retinal vascular tortuosity was resolved nearly three months after the cardiac surgery.

The large percentage of ocular findings in CHD could be related to the high incidence of associated syndromes. ${ }^{4}$ In our study population, chromosome 9 inversion was detected in two patients with pulmonary atresia and Turner syndrome in one with aortic coarctation.

Although this case series investigated the retinal vascular changes in neonatal period in CCHD patients, the study was limited by small number of the patients and as a result may have lacked of the statistical power to detect other ocular pathologies. On the other hand, we could not know about the course of the retinovascular changes in died patients. Nevertheless, to our knowledge, this is the first study investigating retinovascular changes in newborns with CCHD.

\section{CONCLUSION}

CCHD may cause retinovascular changes in newborn patients, although these changes did not require treatment every time. The role of ophtalmologists seems to be more important in this field.

\section{REFERENCES}

1. Huang JB, Liu YL, Lv XD. Pathogenic mechanisms of congenital heart disease. Fetal Pediatr Pathol 2010;29(5):359-72.

2. Strauss A, Toth B, Schwab B, Fuchshuber S, et al. Prenatal diagnosis of congenital heart disease and neonatal outcome--a six years experience. Eur J Med Res 2001;6(2):66-70.

3 Gilboa SM, Salemi JL, Nembhard WN, Fixler DE, et al. Mortality resulting from congenital heart disease among children and adults in the United States, 1999 to 2006. Circulation 2010;122(22):2254-63.

4. Mansour AM, Bitar FF, Traboulsi EI, Kassak KM, et al. Ocular pathology in congenital heart disease. Eye (Lond) 2005;19(1):29-34.

5. Crowe RJ, Kohner EM, Owen SJ, Robinson DM. The retinal vessels in congenital heart disease. Med Biol Illus 1969;19(2):95-9.

6. Tan H, Ceviz N, Baykal O, Büyükavci M, et al. Pulmonary atresia/ventricular septal defect associated with facial portwine stain and retinal vascular abnormality: a new constellation? Am J Med Genet A 2003;122A(3):266-8.

7. Jenkins KJ, Gauvreau K, Newburger JW, Spray TL, et al. Consensus-based method for risk adjustment for surgery for congenital heart disease. J Thorac Cardiovasc Surg 2002;123(1):110-8.

8. Cordina RL, Celermajer DS. Chronic cyanosis and vascular function: implications for patients with cyanotic congenital heart disease. Cardiol Young 2010;20(3):242-53.

9. DeSchweinitzGE, Woods AC. Concerning the ocular symptoms of erythremia (chronic polycythemia vera), with special reference to the fundus picture. Trans Am Ophthalmol Soc 1925;23:90-105.

10. Tsui I, Shamsa K, Perloff JK, Lee E, et al. Retinal vascular patterns in adults with cyanotic congenital heart disease. Semin Ophthalmol 2009;24(6):262-5.

11. Ahmad OF, Hirose T. Severe retinopathy in a child with hypoplastic left heart syndrome. Am J Ophthalmol 2004;137(3):566-7.

12. el-Asrar AM, Awad A, Tabbara KF. Retinal arterial macroaneurysm in a patient with congenital heart disease. Br J Ophthalmol 1993;77(9):606-7.

13. Section on Ophthalmology American Academy of Pediatrics; American Academy of Ophthalmology; American Association for Pediatric Ophthalmology and Strabismus. Screening examination of premature infants for retinopathy of prematurity. Pediatrics 2006; 117(2):572-6.

14. Petersen RA, Rosenthal A. Retinopathy and papilledema in cyanotic heart disease. Pediatrics 1972;49(2):243-9. 